\title{
Correction to: Minimal residual disease- and graft-vs.-host disease-guided multiple consolidation chemotherapy and donor lymphocyte infusion prevent second acute leukemia relapse after allotransplant
}

Chen-Hua Yan ${ }^{1,2}$, Yu Wang ${ }^{1,2}$, Jing-Zhi Wang ${ }^{1}$, Yu-Hong Chen ${ }^{1}$, Yao Chen ${ }^{1}$, Feng-rong Wang ${ }^{1}$, Yu-Qian Sun ${ }^{1}$, Xiao-Dong Mo', Wei Han ${ }^{1}$, Huan Chen ${ }^{1}$, Xiao-hui Zhang ${ }^{1,2}$, Lan-Ping Xu' ${ }^{1}$, Kai-Yan Liu ${ }^{1,2}$ and Xiao-Jun Huang ${ }^{1,2^{*}}$

Correction to: J Hematol Oncol (2016) 9:87 https://doi.org/10.1186/s13045-016-0319-5

The original article [1] contains two errors in the MRD Testing sub-section of the Methods:

1) The description, “...by four-color flow cytometry (FCM)" was incorrect. The correct description should be "...by eight-color flow cytometry (FCM)".

2) The description, "LAIPs were detected by fourcolor FCM" was incorrect. The correct description should be "LAIPs were detected by eight-color FCM".

Received: 26 February 2019 Accepted: 27 February 2019 Published online: 05 March 2019

\section{Reference}

1. Yan $\mathrm{C}-\mathrm{H}$, et al. Minimal residual disease- and graft-vs.-host disease-guided multiple consolidation chemotherapy and donor lymphocyte infusion prevent second acute leukemia relapse after allotransplant. J Hematol Oncol. 2016;9:87. https://doi.org/10.1186/s13045-016-0319-5.

\footnotetext{
* Correspondence: huangxiaojun@bjmu.edu.cn

'Beijing Key Laboratory of Hematopoietic Stem Cell Transplantation, Peking University Institute of Hematology, Peking University People's Hospital, Xi Zhimen South Street No. 11, Beijing 100044, China

${ }^{2}$ Collaborative Innovation Center of Hematology, Xi Zhimen South Street No.

11, Beijing 100044, China
} 